\title{
Narrative review: indeterminate pulmonary nodules
}

\author{
Benjamin Wadowski^, Raphael Bueno \\ Division of Thoracic Surgery, Brigham and Women's Hospital and Harvard Medical School, Boston, MA, USA \\ Contributions: (I) Conception and design: Both authors; (II) Administrative support: R Bueno; (III) Provision of study materials or patients: None; (IV) \\ Collection and assembly of data: B Wadowski; (V) Data analysis and interpretation: Both authors; (VI) Manuscript writing: Both authors; (VII) Final \\ approval of manuscript: Both authors. \\ Correspondence to: Dr. Raphael Bueno, MD. Chief, Division of Thoracic Surgery, Brigham and Women's Hospital. 75 Francis Street, Boston, MA \\ 02115, USA. Email: rbueno@bwh.harvard.edu.
}

\begin{abstract}
The detection of indeterminate pulmonary nodules (IPNs) has become common through more frequent use of sensitive axial imaging and implementation of lung cancer screening protocols. Differentiating benign and malignant lesions can be challenging, particularly when nodules are small, but presents a critical opportunity to detect early cancers and improve patient outcomes. There are many noninvasive and invasive approaches to characterizing IPNs, representing an ongoing clinical need and an area of active research. This article will first provide a brief overview of the epidemiology of IPNs and discuss the implications of lung cancer screening. It will then review the use of clinical scores, radiographic features, and noninvasive molecular approaches in risk stratifying IPNs with an emphasis on sub-centimeter lesions. In particular, the evidence underlying peripherally accessible DNA, RNA, and protein-based "liquid biopsies" will be explored. This article will then describe techniques to optimize the diagnostic yield of minimally invasive tissue sampling methods including percutaneous transthoracic needle biopsies and endobronchial ultrasound-guided fine needle aspiration, as well as approaches to optimize lesion localization for surgical diagnosis. The review will conclude with a discussion of several molecular tests that provide insight into possible ways to overcome histologically indeterminate or nondiagnostic biopsy results in the future.
\end{abstract}

Keywords: Nodule; indeterminate; screening; molecular; pulmonary

Received: 05 June 2020; Accepted: 03 November 2020; Published: 25 November 2022.

doi: $10.21037 /$ ccts-20-113

View this article at: http://dx.doi.org/10.21037/ccts-20-113

\section{Introduction}

Detecting lung cancer at an early stage can significantly prolong survival (1), but it is often insidious and the majority of patients who present with symptoms harbor advanced disease (2). To maximize early detection and prolong survival, there is a critical need to address the possibility of malignancy in all patients with either screen-detected or incidental indeterminate pulmonary nodules (IPN). An IPN can be defined as an opacity in the lung that measures less than $3 \mathrm{~cm}$, is completely surrounded by pulmonary parenchyma, and lacks features that strongly suggest a benign etiology $(3,4)$.
Although the majority of IPNs represent benign disease, their management presents a significant challenge with a cost of up to $\$ 28$ billion/year in the U.S. alone (5). In particular, differentiating malignant from benign IPNs has been the subject of a great deal of ongoing research.

This article will provide a brief overview of the epidemiology of IPNs. Next, it will review clinical, radiographic, and molecular approaches to predicting the likelihood of malignancy among IPNs with an emphasis on small $(<1 \mathrm{~cm})$ lesions. It will also address technical innovations to enhance the diagnostic yield of invasive procedures and optimize the success of surgical resection.

^ ORCID: 0000-0003-4949-821X. 
The objective of this review is to provide clinicians with an understanding of the current resources available to risk-stratify IPNs without or prior to obtaining surgical diagnosis. We present the following article in accordance with the Narrative Review reporting checklist (available at https://ccts.amegroups.com/article/view/10.21037/ccts-20$113 / \mathrm{rc})$.

\section{Methods}

For this narrative overview, a MEDLINE search of English-language articles from 1/1/2,000 through present matching the key words "indeterminate pulmonary nodule" or "lung cancer screening" was performed. Additional hand searches of the references of retrieved literature were performed. Emphasis was placed on controlled trials from 2015 through present with earlier publications included for additional historical context.

\section{Background and epidemiology}

Small IPNs are identified in one of two contexts: through screening protocols, or as incidental findings. Lung cancer screening has been widely implemented for at-risk patient populations to identify disease at an earlier stage. The National Lung Screening Trial (NLST), changed the paradigm for lung cancer screening in 2011 by demonstrating a $20 \%$ relative reduction in lung cancer mortality and a $6.7 \%$ reduction in all-cause mortality using annual low-dose CT (LDCT) in high risk patients (6). The benefits of screening in mortality reduction have been re-demonstrated in several other large trials $(7,8)$. However, screening comes at the cost of false positive results and the additional testing, including invasive procedures, necessary to manage indeterminate findings. For example, over the course of the NLST nearly $40 \%$ of subjects had at least one positive screening test, with a false positive rate for each round ranging from $16.8-27 \%$ (6). In another screening study, the proportion of indeterminate CT scans was $19.7 \%$ at baseline (7).

Although false positive screening rates have been reduced over time $(7,8)$, they constitute a major challenge. In an analysis of the Pan-Canadian Early Detection of Lung Cancer Study (PanCan) and the British Columbia Cancer Agency database, malignancy rates among patients with screen-detected nodules were $5.5 \%$ and $3.7 \%$ respectively (9). Even among IPNs that are concerning enough to warrant surgical evaluation, the frequency of benign disease varies widely (e.g., 10-55\%) (4). For example, in the PanCan study, 16/137 (12\%) of surgical resections yielded benign lesions (10). It is critical to note, however, that these studies are constrained within a limited period of follow up. Study periods vary: The NLST, for example, had a median follow-up of approximately 6.5 years, while several others [e.g., Lung Screening Study (11), German Lung Cancer Screening Intervention Trial (12)] are limited to 1-3 years. Therefore, the malignancy rates identified in these studies likely underestimate the rates of lifetime detection for subjects at risk.

Outside of screening, the detection of incidental IPNs has increased along with trends in use of axial imaging: between 2006 and 2012 the annual rate of having at least one positive CT scan increased from 3.9 to 6.6 per 1,000 person-years by one analysis, yet the rate of new lung cancer diagnosis within 2 years of index scan remained unchanged (13). Notably, in this study $45 \%$ of the largest identified nodules were $8 \mathrm{~mm}$ or smaller. The prevalence of IPNs has led to the implementation of dedicated infrastructure for surveillance and diagnosis, although the utility of these efforts is unclear. In a cohort of 414 patients followed at a dedicated clinic for surveillance of indeterminate nodules, for example, the median initial nodule size was $6 \mathrm{~mm}$ (14). While $10.1 \%$ of these patients underwent fluorodeoxyglucose (FDG)positron emission tomography (PET) imaging and $4.8 \%$ required invasive testing or surgery, only $3 \%$ ultimately received a diagnosis of cancer.

Invasive testing to differentiate true from false positives can be costly and psychologically burdensome for patients, but despite inherent procedural risks the overall morbidity is low. For example, the total incidence of major complications in the LDCT arm of the NLST was $1.4 \%$; these were much more frequent in the true positive group than in the false positive group (11.2\% vs. $0.06 \%)$, and most were related to surgical procedures rather than minimally invasive biopsy techniques $(6,15)$. Only $1.2 \%$ and $0.7 \%$ of patients with benign lesions in the NLST underwent needle biopsy/bronchoscopy or surgery, respectively. These rates were similar to those in the NELSON study (1.2\% and $0.6 \%$, respectively). In an analysis of the NLST data by Bach and colleagues, the risk of death and major complication after any diagnostic testing (including nonscreening imaging) in patients with benign nodules was 4.1 and $4.5 / 10,000$, respectively (16). For invasive procedures in particular, the risk of death within 2 months was $6 / 10,000$ in the whole cohort; this was reduced to $2.2 / 10,000$ when restricted to patients who were ultimately found to have benign nodules (17). 
Table 1 Risk of lung cancer by nodule size

\begin{tabular}{lc}
\hline Size $(\mathrm{mm})^{\dagger}$ & Cancer \% \\
\hline NSLT $(23)^{\ddagger}$ & 0 \\
$<4$ & 0.5 \\
$4-6$ & 1.7 \\
$7-10$ & 11.9 \\
$11-20$ & 29.7 \\
$21-30$ & 41.3 \\
$>30$ & \\
NELSON $(21)^{\S}$ & 0.4 \\
$<5$ & 1.3 \\
$5-10$ & 11.1 \\
$10-<15$ & 19.6 \\
$15-<20$ & 25 \\
$20-<30$ & 31.6 \\
$\geq 30$ &
\end{tabular}

${ }^{\dagger}$ Refers to diameter of the largest nodule detected on screening examination; ${ }^{\ddagger}$ Represents the proportion of nodules confirmed to be lung cancer during the NLST study period; ${ }^{\S}$ Represents the probability of lung cancer diagnosis in the NELSON cohort within 2 years of screening test.

On balance, therefore, the clinical harms of invasive diagnostic testing are modest and appear to be outweighed by the significant reduction in lung cancer mortality provided through screening and proactive management of IPNs. The challenge has therefore shifted from merely detecting patients with pulmonary lesions to accurately predicting which IPNs are high-risk and warrant further treatment.

\section{Radiographic risk stratification-the first line}

Upon detection, IPNs are characterized in terms of size, morphology (i.e., solid, part-solid, or ground glass), and distribution. Each of these features has independent implications for malignancy risk. Extensive research into advanced radiographic analytics, or radiomics, has also augmented the predictive ability of descriptive traits alone.

Size is perhaps the single greatest radiographic predictor of malignancy risk, as the prevalence of malignancy increases significantly with larger nodule size $(9,18,19)$. The risk of malignancy is generally considered $<1 \%$ for nodules $<5 \mathrm{~mm}$, and in the NELSON study the malignancy risk for subjects with nodules $<5 \mathrm{~mm}$ was similar to the risk in subjects without nodules $(20,21)$. In the same study, nodules $5-10 \mathrm{~mm}$ in diameter had a $1.3 \%$ prevalence of malignancy, while those $>10 \mathrm{~mm}$ had a $15.2 \%$ prevalence of malignancy (22). These findings have been corroborated in other large series. A comparison of malignancy risk by nodule size between the NLST and the NELSON cohorts is shown in Table 1 (21,23).

Size by size, solid, subsolid, and ground glass nodules may follow different management algorithms. While a full exploration of these distinctions is out of the scope of this review, Sanchez, Benegas, and Vollmer studied differences in guidelines by morphology for incidental nodules $<8 \mathrm{~mm}$ (24). In general, small subsolid nodules are associated with a higher risk of malignancy than similarly sized solid nodules and require closer follow-up. In addition, there is a significant correlation between size of the solid component of subsolid nodules and the invasive component on pathology (25). The authors note that patient-specific cancer risk (discussed below) should also be taken into account for small nodules to determine next steps in management. If a patient is deemed high risk $(>10 \%)$, they suggest consideration of positron emission tomographycomputed tomography (PET-CT) despite the low utility of this modality in nodules $<8 \mathrm{~mm}$. Notably, $\mathrm{O}$ and colleagues found that subcentimeter nodules with no FDG uptake in oncologic patients were still malignant in 19\% of cases (26). Therefore, high risk oncologic patients may warrant biopsy even in the context of a negative PET-CT.

Other static radiographic features have been associated with risk of malignancy. For example, malignant nodules tend to be surrounded by significantly more vessels than benign nodules, and the area under curve (AUC) for vessel count alone was $>0.7$ in one model (27). Marginal spiculation, degree of enhancement (by Hounsfeld units, $\mathrm{HU})$, and pleural indentation were also found to be independent predictors of malignancy (28). Indeed, margin characteristics (smooth, lobulated, or spiculated) are consistently correlated with malignancy risk (29). Lack of calcification increases long-term lung cancer risk ratios at the person, lung, and lobe levels (5).

Advanced computational techniques have been incorporated into these analyses as well. For example, semi-automated region-of-interest selection can assist in discerning malignant from benign lesions among indeterminate pulmonary nodules. In one study of 90 pathologically confirmed nodules of which 42 (46.7\%) 
were malignant, semi-automated region-of-interest selection identified significantly higher mean attenuation of enhancement following contrast administration in malignant nodules. A cut-off value of $15 \mathrm{HU}$ change yielded $83 \%$ sensitivity, $65 \%$ specificity, and $73 \%$ accuracy for predicting malignancy (30). Li and colleagues developed a machine learning algorithm applying custom quantitative imaging "biomarkers" to classify ground glass nodules (31). The algorithm was able to distinguish adenocarcinoma in situ or minimally invasive adenocarcinoma with greater accuracy than three thoracic radiologists $(88.1 \%$ vs. $60.8 \%$ respectively), without the need for contrast enhancement.

Beyond static imaging characteristics, growth rate is a major radiographic indicator of malignancy in nodules $<8$ $\mathrm{mm}$. There are multiple approaches to assessing growth rate, including two-dimensional sizing and volumetry, the latter of which is favored by the British Thoracic Society guidelines for incidental pulmonary nodules (32). Borghesi and colleagues explored the role of computer-aided assessment of volume doubling time (VDT) in nodules within this size range, comparing 400 and 600-day time cutoffs (33). They found that, unsurprisingly, a 600-day VDT cutoff reduced the number of false negatives for solid nodules compared to a 400-day VDT. In subsolid nodules, change in computer-defined radiographic features (such as entropy, skewness, and compactness) over time strongly suggests malignancy as well (34).

Nuclear imaging is often used in staging suspected pulmonary malignancy, but may be unreliable for small IPNs (26). In one study, FDG PET/CT was assessed as an adjunct to LDCT in distinguishing benign from malignant lesions among indeterminate lung nodules (19). While selective FDG PET/CT accurately characterized indeterminate nodules detected on screening LDCT overall, sensitivity decreased substantially to $25 \%$ for incidence nodules $<10 \mathrm{~mm}$. PET/CT scanning using ${ }^{68} \mathrm{Ga}-$ DOTATATE has also been investigated in this context (35). In a small study of 31 lesions in 30 patients, DOTATATE was more specific (94\% vs. $81 \%$ ) but less sensitive $(73 \%$ vs. 93\%) than FDG. DOTATATE uptake was correlated with SSTR2A expression by immunohistochemistry in the stroma (but not tumor cells) of $55 \%$ of non-small cell lung cancer (NSCLC). Overall, however, accuracy of diagnosis was equivalent between the two methodologies.

\section{Clinical risk scores}

Radiographic features are often combined with patient- specific clinical characteristics to derive overall risk scores and guide management. A variety of clinical predictors to risk-stratify solitary pulmonary nodules are available, but all differ slightly, lack wide external validation, and have varying degrees of success when applied to a common cohort of nodules (36). However, certain key features are conserved and worth noting. For example, Swensen and colleagues identified that older age, smoking history, and history of extra-thoracic cancer independently increase risk of malignancy along with larger nodule size, upper lobe location, and spiculation (37). More recently, McWilliams and colleagues developed tools incorporating older age, female sex, family history of lung cancer, and emphysema with upper lobe location, subsolid morphology, lower nodule count, and spiculation as predictors of cancer (9). Patient history of antecedent cancer, both pulmonary and extrathoracic, can complicate these assessments. Mery and colleagues studied 1,104 patients with pulmonary nodules by cancer history and found that prior extrapulmonary malignancy increased the risk of cancer in a given nodule from $63 \%$ to $79 \%$, with $52 \%$ of these cancers being lung and $48 \%$ being metastases (38). Notably, the histology of the previous tumor was impactful with tumors such as melanoma, sarcoma, renal cancer, and gastrointestinal cancers having the highest metastatic potential. In the same study, $82 \%$ of nodules in patients with a history of lung cancer were malignant, compared to $63 \%$ in the study population as a whole.

It may be helpful to aggregate key validated risk factors and present clinicians with risk estimates based on multiple models simultaneously. For example, the American Association for Thoracic Surgery has published the Lung Cancer Risk Assessment Tool (https://www.aats.org/ aatsimis/AATSWeb/Association/About/Resources/Lung Cancer_Risk_Assessment_Tool), which incorporates age, sex, BMI, smoking history, family history, prior chest $\mathrm{x}$-rays, prior malignancy, pneumonia, emphysema, and asbestos exposure to provide four different risk estimates (39).

Given the widespread availability of these scores, the 2007 American College of Chest Physicians guidelines (40) recommended documentation of the pretest probability of malignancy for all solitary pulmonary nodules. However, clinicians often rely on qualitative impressions in making management decisions. Maiga and colleagues found that that fewer than $5 \%$ of patients with lung nodules have a documented quantitative prediction of malignancy prior to tissue diagnosis (41). Those without a quantitative score had a qualitative risk statement documented $63.1-78.1 \%$ 
of the time. Interestingly, when qualitative statements were categorized in bins and ranked, the authors found a significant correlation between strength of the statement and higher Mayo Clinic Model-predicted probability of cancer (41).

Therefore, while the predictors of malignancy are fairly well described, the ways in which clinicians use these factors in determining management strategy for IPNs is less clear. In a quantitative study assessing the impact of different clinical factors on decision making by 153 pulmonologists, the authors found that invasive testing was more likely to be pursued in patients with younger age, higher smoking history, and larger nodules (4). Invasive testing was less likely to be recommended for older patients even when clinicians were informed that all patients were good surgical candidates. Despite the qualitative nature of management decisions based on clinical characteristics, strikingly, the addition of a hypothetical biomarker test result had a strong and independent effect on management decisions (4). This suggests a need for more quantitative data upon which to base risk assessments in IPNs.

\section{Molecular biomarkers}

While the clinical algorithm for management of larger nodules is predicated on a high chance of malignancy, smaller nodules are well suited to adjunctive methods for risk stratification given a lack of consensus on optimal management (42). There is significant value in the development of molecular tests to enhance the accuracy of risk stratification prior to lesion sampling. These biomarkers, sometimes referred to as "liquid biopsies", can be derived from easily accessible tissues (e.g., saliva, plasma, serum), and encompass a broad range of targets.

Micro-RNAs in particular are attractive substrates for the development of liquid biopsies, as they are highly stable in plasma (43). Lin and colleagues investigated the use of a classifier combining clinical/radiological characteristics with plasma expression of 11 miRNAs in former smokers with IPNs (44). The classifier incorporated two of the examined 11 (mi-126 and mi-205-5p) resulting in an AUC of 0.94 with a sensitivity and specificity of $88.9 \%$ and $90.5 \%$, respectively, for a diagnosis of malignancy. This combined classifier was superior either the biomarker panel or a clinical/radiographic risk model alone. Xing and colleagues studied a sputum miRNA panel in preoperative identification of malignant nodules (45). They quantified the expression of 13 miRNAs which had previously been identified as signatures of lung cancer: miRs $21,31,126$, $143,155,182,200 \mathrm{~b}, 205,210,372,375,486$, and 708. Of note, five of these $(21,126,200,205,210)$ overlap with the plasma panel described above (44). A panel of three miRNA biomarkers (miR21, 31, and 210) produced sensitivity and specificity for identifying malignant solitary nodules in excess of $80 \%$ in two independent test cohorts, performing significantly better than sputum cytology in terms of sensitivity ( $82.9 \%$ vs. $44.3 \%)$.

$\mathrm{Ma}$ and colleagues identified a pair of peripheral blood mononuclear cell (PBMC)-miRNA biomarkers (miRs-19b$3 p$ and $-29 b-3 p$ ) which were shown to predict malignancy with $72.6 \%$ sensitivity and $82.6 \%$ specificity when overexpressed (46). They then tested these biomarkers in combination with smoking pack-years and spiculation to develop a cancer prediction score for indeterminate nodules with an AUC of 0.91 at 80.4 and $89.1 \%$ sensitivity and specificity, respectively (47). Interestingly, while this model performed poorly in distinguishing among different stages of NSCLC, it could identify small cell carcinoma with higher specificity compared to other types of cancer.

Protein-based assays of peripheral samples have been investigated. Differential expression of markers of angiogenesis (HB-EGF, EGF, VEGF-A, VEGF-C, and VEGF-D, follistatin, PLFG, and BMP-9) in serum was retrospectively shown to improve discrimination between benign nodules and stage I NSCLC (48). Li and colleagues developed a 13-protein plasma-based test based on a discovery set of 143 nodules with known pathology (49). They validated this classifier, which was agnostic to clinical and radiographic features, in an independent set of 104 plasma samples, yielding a negative predictive value of $90 \%$. However, the efficacy of this classifier was not examined in subgroup analysis of nodules $<10 \mathrm{~mm}$ in size. The use of this classifier was further explored in a multicenter study of patients with indeterminate nodules undergoing invasive diagnostic procedures using a prospective collection/ retrospective-blinded-evaluation approach (50). Using the classifier, the authors found that $32 \%$ of surgeries and $31.8 \%$ of overall invasive procedures on benign nodules could have been avoided. However, up to $24 \%$ of patients with malignancy would have been incorrectly assigned to CT surveillance.

Antibody-based biomarkers have also been explored. Lastwika and colleagues isolated a set of autoantibodies from tumor-infiltrating B cells in NSCLC and matched them to known cancer antigens using a protein screen (51). They found that autoantibodies in NSCLC are not 
only found in peripheral plasma but are also specific for malignant nodules. Five autoantibody targets were confirmed to be significantly higher in patients with malignant nodules than in control patients. Finally, the authors developed a four-marker panel (FCGR2A, EPB41L3, and LINGO1 IgG-complexed autoantibodies as well as S100A7L2 IgM-complexed autoantibody) which had an AUC of 0.737 (at $33.3 \%$ sensitivity, 90\% specificity). In patients with indeterminate pulmonary nodules $8-20 \mathrm{~mm}$, the AUC of this panel improved to 0.78 (91.7\% sensitivity and $75.1 \%$ specificity). Massion and colleagues analyzed the utility of the Early CDT-Lung blood test, which measures seven tumor-associated antigens, in discriminating benign from malignant nodules (42). Particularly in nodules $4-20 \mathrm{~mm}$, a positive test result correlated with a significantly increased risk of malignancy compared to nodule size alone. A combination of binary tests improved the discriminatory ability of the overall panel. However, a negative test result did not reliably obviate the need for ongoing monitoring of IPNs (42).

DNA-based molecular tests are also under investigation and hold promise for both minimally invasive detection and tumor characterization (52). Tailor and colleagues performed a retrospective study using whole exome sequencing of plasma cell free DNA with matched PBMC germline DNA in 16 patients with benign nodules and 17 patients with malignant nodules (53). They identified 10 variants observed in at least two cancer patients each, but not in control patients. Chen and colleagues evaluated plasma from 163 cases of Stage IA or IB NSCLC for promoter methylation of 8 lung cancer-specific genes (CDO1, TAC1, SOX17, HOXA7, HOXA9, GATA4, GATA5, and PAX5) using nanoparticle-based DNA extraction followed by quantitative methylation-specific PCR (54). Compared to 83 benign controls, plasma methylation for CDO1, TAC1, SOX17, and HOXA7 was significantly higher in cancer. A three-gene combination yielded sensitivity and specificity of $90 \%$ and $71 \%$ respectively, with an AUC of 0.88 . These tests were effective even tumors $<1 \mathrm{~cm}$ (54).

One limitation of molecular approaches to classifying subcentimeter lesions is that most molecular tests are derived, by necessity, from training sets of large or advanced-stage tumors. The thresholds and biomarkers identified, therefore, may not be applicable for small or early-stage tumors. For example, pathologic tumor size has been shown to correlate with mean plasma variant allele frequencies of single copy number variants, and detection of circulating tumor DNA varies by lymph node status and tumor size (55). In addition, few sets of small lesions with known histology are available for validation. Finally, there is often a lack of clarity as to whether tests would be more appropriately used for screening or diagnostic purposes.

\section{Minimally invasive methods to biopsy IPNs}

If clinical, radiographic, and molecular risk stratification algorithms remain inconclusive or suggest malignancy, tissue sampling of the IPN is warranted. Strategies to obtain tissue include percutaneous transthoracic needle biopsies (PTNBx), endobronchial ultrasound-guided fine needle aspiration (EBUS-FNA), and surgical resection.

Although biopsy is often seen as a definitive diagnostic step, nonspecific results are not uncommon. In a review of 226 lesions initially classified as "nonspecific benign" on transthoracic needle biopsy, 24 (10.6\%) were eventually diagnosed as malignant based on radiological or pathological follow-up (56). Subsolid nodules were associated with higher odds of false-negative biopsy (OR 3.95), while granulomatous inflammation and documentation of needle tip within target were associated with lower odds of false negative biopsy (OR 0.04 and 0.37 , respectively). Further, Witt and colleagues studied 50 cases of NSCLC diagnosed by FNA with concurrent core biopsy or resection as a reference standard (57). They found that interobserver agreement in classifying cases as squamous cell or adenocarcinoma was poor at 0.22 when an indeterminate category was included and 0.1 when it was not, with overall accuracy for differentiating these two pathologies at $65 \%$ and $66 \%$ respectively. This suggests that cytomorphology alone is often insufficient to subclassify NSCLC. In another study of 52 CT-guided core needle biopsies (CNBx) for subsolid nodules, 4 (7.7\%) yielded indeterminate pathology, and $4(7.7 \%)$ were false negatives (58). Of 17 cases with surgical correlation, diagnostic yield for cancer and diagnostic accuracy of $\mathrm{CNBx}$ were both $82.4 \%$.

Several factors have been identified which increase the diagnostic yield of PTNB. Diagnostic results are more likely in larger lesions, solid lesions, the use of cutting or combined cutting/aspiration biopsies $v s$. aspiration alone, increasing the number of samplings, and use of a coaxial needle (59).

For endobronchial sampling, Layfield, Dodd, \& Witt examined results from 155 EBUS-FNAs with surgical follow up (60). Cytolopathologic results were categorized as non-diagnostic, benign, atypical, suspicious and malignant. After resection, $40 \%$ of the non-diagnostic and $24 \%$ of 
benign biopsies were diagnosed as malignant. In contrast, $87 \%$ of malignant lesions on cytopathology were confirmed malignant after resection. This suggests that EBUS-FNA, while fairly specific, may provide false assurance of the benignity of a given lesion in a significant proportion of cases. Peripheral pulmonary lesions with a bronchus sign are more likely to be successfully diagnosed with guided bronchoscopy than those without a bronchus sign (61).

Subcentimeter nodules present a unique challenge for transthoracic tissue sampling. While diagnostic accuracy for nodules $>1 \mathrm{~cm}$ is generally excellent, estimates for smaller nodules range from $52-88 \%$ (62). In addition, these lesions have relatively high rates of complications following biopsy such as pneumothorax (up to $62 \%$ in one study) and requirement for thoracostomy tube insertions (up to 9.1\%) $(62,63)$. Fluoroscopic guidance is the traditional method for PTNBx but has disadvantages with respect to small nodules such as inability to visualize key vascular structures in the needle path and difficulty pinpointing small nodules in orthogonal planes (64). $\mathrm{Ng}$ and colleagues found that CTguided percutaneous FNA in a small series of 47 lesions had an overall sensitivity, specificity, and accuracy of $67.7 \%$, $100 \%$, and $78.8 \%$ respectively (62). C-arm cone-beam CT with or without virtual navigation guidance has also been studied and performs comparably well for $<10 \mathrm{~mm}$ nodules as for $10-20 \mathrm{~mm}$ nodules, although indeterminate pathology remains prevalent (e.g., 8.4\%) $(64,65)$.

\section{Intraoperative localization strategies}

When surgical diagnosis is necessary, IPNs should generally be treated by wedge resection with intraoperative histological analysis $(66,67)$. Unfortunately, diagnostic uncertainty may persist even after resection has been attempted. Small nodules, particularly those deep within the lung parenchyma, can be difficult to localize. A variety of approaches have been studied to optimize the diagnostic yield of wedge resection and avoid missed lesions or indeterminate diagnoses.

Localization techniques for small nodules include tattooing and image-guided wire placement, as thoracoscopic palpation alone is most effective for nodules located in the outermost portion of the lung (68). Ko and colleagues studied the use of low-dose patent blue dye injection for lesion localization in 137 IPNs with mean size $9.5 \mathrm{~mm}$ (69). While $40 \%$ developed pneumothorax and $12.8 \%$ developed focal parenchymal hemorrhage, zero required chest tube placement. Localization was successful in $98.5 \%$.
While patent blue diffuses less than methylene blue, dyebased methods may be difficult to implement for deeper nodules. Instead, radiolocalization can be a useful adjunct to minimally invasive resection. Manca et al. (70) (2018) reviewed 395 patients with small nodules $(<2 \mathrm{~cm})$ more than $5 \mathrm{~mm}$ below visceral pleural who underwent CT-guided radiolocalization prior to video-assisted thoracoscopic surgical (VATS) resection. They found that average time for localization was just three minutes with no major perioperative complications or mortalities and reported a $99 \%$ VATS success rate for radioguidance with a $1 \%$ open conversion rate for difficulty localizing the hot area.

In contrast to staged localization and resection, work from our institution has described an approach to VATS using real-time intraoperative CT-guidance (iVATS) (71). In a hybrid operating room under general anesthesia, patients are placed in lateral decubitus position and undergo C-arm CT scan of the region of chest containing the target lesion. These images are then used to guide percutaneous placement of fiducials using T-bars under fluoroscopy for immediate VATS localization. In a prospective clinical trial, the safety and technical success of this technique was demonstrated for relatively small lesions $(0.6-1.8 \mathrm{~cm})$ with no major intraoperative or postoperative complications or mortality (71). These findings were later validated in a larger cohort of patients following broader implementation into clinical practice, with successful resection of $97 \%$ of nodules (72). Yu and colleagues (2018) later described a different hybrid room approach using real-time imageguided localization to reduce the time interval between hookwire placement and resection in patients with mean nodule size $\sim 9.1 \mathrm{~mm}$ (73). They demonstrated a shorter "at-risk" period between placement and resection (109.5 minutes on average vs. 41.1 minutes for imageguided VATS, $\mathrm{P}=0.011$ ), as well as lower risk of hookwire dislodgement ( $25 \%$ vs. $0 \%, \mathrm{P}=0.036$ ).

Some lesions, particularly deep or central nodules, are difficult to access for wedge resection to establish a diagnosis. Isaka and colleagues studied the safety and efficacy of VATS core needle biopsy for indeterminate tumors, finding a sensitivity, specificity, and accuracy of $94.3 \%, 87.5 \%$, and $93.7 \%$ respectively with no complications and no pleural dissemination in the experimental group (74). However, tumors in this study were fairly large at $>2 \mathrm{~cm}$ on average.

Beyond localization, molecular and imaging techniques can be integrated to predict nodule malignancy intraoperatively. In a prospective pilot study of 30 patients 
with indeterminate pulmonary nodules, Kennedy and colleagues studied the utility of "optical biopsy" or nonradioactive intraoperative molecular imaging in predicting whether a nodule was adenocarcinoma (75). They found that the test can be performed rapidly, with a positive predictive value of $100 \%$ for undergoing pulmonary lobectomy with no false positives or negatives; of 19 positive optical biopsies, 18 were positive on frozen section and underwent lobectomy while 1 was frozen-negative.

\section{The pathology indeterminate nodule: features and approaches}

Surgeons may be placed in the difficult situation of receiving indeterminate pathology reports, either from nonsurgical biopsies or, rarely, from resection specimens. When sampled tissue sent for intraoperative frozen pathologic analysis is found to be indeterminate, additional tissue can be taken at that time. If the concern for malignancy is high, based on preoperative factors, a more substantial resection can be performed such as a lobectomy if the initial resection was a wedge resection.

In a large, multicenter retrospective analysis Lee and colleagues examined the malignancy risk of lung lesions with nondiagnostic PTNB results (59). Nondiagnostic PTNBs fell into three categories: nonspecific benign pathologic findings, atypical cells, and insufficient specimen. The authors analyzed 9,384 biopsies in 9,239 patients, of which 2,590 (27.6\%) were considered non-diagnostic. Of nondiagnostic biopsies, the malignancy rates of nonspecific benign, atypical cells, and insufficient samples were $21.3 \%$, $90.1 \%$, and $46.6 \%$ respectively. Among these patients, 335 patients underwent repeat PTNB yielding 53.7\% diagnostic results. A diagnostic result on second attempt was most likely when the initial report was "atypical cells" at $63 \%$. Based on these findings, the authors developed a management algorithm that recommends surgical biopsy in the case of atypical cells suspicious or suggestive for malignancy, as well as in clinically high-risk patients with "insufficient specimen" on initial biopsy.

Postoperatively, molecular techniques can lend clarity to lesions with indeterminate pathology on biopsy. Murphy and colleagues studied the use of somatic DNA junction breakpoints from genomic rearrangements to distinguish primary lung cancer from pulmonary metastases given their highly specific nature (76). They performed matepair sequencing on DNA from 76 distinct tumors in 37 patients and were able to classify lineage in all tumor pairs, while histologic review misclassified lineage in 27\% of same-histology pair comparisons. Gordon and colleagues developed a ratio-based test using 23 genes to differentiate normal lung, small cell cancer, NSCLC, and carcinoid tumors with $100 \%$ accuracy from FNA (77). Later, De Rienzo and colleagues developed a gene-ratio algorithm using only four genes (AGER, GPR 56, SSP1, DDR1) to reliably distinguish normal lung from NSCLC using a minimal amount of nucleic acid material from FNA (78). However, given their reliance on relatively unstable mRNA, these tests may be difficult to implement outside of academic centers. In contrast, Christensen and colleagues studied CpG methylation profiles in biopsy specimens from NSCLC as well as normal pleura and mesothelioma (79). They were able to differentiate among the three different sample types using unsupervised modeling techniques based on methylation profiles alone with high accuracy, particularly in distinguishing adenocarcinoma from mesothelioma ( $>99 \%)$.

\section{Conclusion}

Indeterminate pulmonary nodules have become common with more frequent use of sensitive axial chest imaging and widespread implementation of screening protocols. Determining the malignant potential of IPNs, particularly those under $1 \mathrm{~cm}$, represents a unique diagnostic challenge for clinicians and a critical effort to protect patients from lung cancer, the leading cause of cancer-related diagnosis and death worldwide. Clinical, radiographic, and molecular approaches have been developed to risk-stratify these lesions in order to maximize patient benefit and minimize harm. In addition, several different techniques have been described to enhance the accuracy and diagnostic yield of minimally invasive tissue sampling methods. Truly indeterminate pathologic results are rare following surgical resection of IPNs, and these unique circumstances warrant multidisciplinary evaluation and case-by-case consideration of treatment strategy.

\section{Acknowledgments}

Funding: None.

\section{Footnote}

Provenance and Peer Review: This article was commissioned by the Guest Editors (Scott Swanson, Daniel Dolan) for 
the series "How to Evaluate, Diagnose and Treat Small Lung Nodules" published in Current Challenges in Thoracic Surgery. The article has undergone external peer review.

Reporting Checklist: The authors have completed the Narrative Review reporting checklist. Available at https:// ccts.amegroups.com/article/view/10.21037/ccts-20-113/rc

Peer Review File: Available at https://ccts.amegroups.com/ article/view/10.21037/ccts-20-113/prf

Conflicts of Interest: Both authors have completed the ICMJE uniform disclosure form (available at https://ccts. amegroups.com/article/view/10.21037/ccts-20-113/coif). The series "How to Evaluate, Diagnose and Treat Small Lung Nodules" was commissioned by the editorial office without any funding or sponsorship. RB reports grants from Medgenome, grants from Roche, grants from Verastem, grants from Merck, grants from Gritstone, grants from Epizyme, grants from Siemens, grants from Celsisus, grants from NCI, grants from DoD, grants from NIH, grants from Genetech, outside the submitted work; In addition, RB has a patent $7,622,260$ licensed to $\mathrm{BWH}$, a patent $8,450,057$ licensed to $\mathrm{BWH}$, a patent $8,551,700$ licensed to $\mathrm{BWH}$, and a patent 9,446,050 licensed to BWH and Patents/Equity in Navigation Sciences. Both authors have no other conflicts of interest to declare.

Ethical Statement: The authors are accountable for all aspects of the work in ensuring that questions related to the accuracy or integrity of any part of the work are appropriately investigated and resolved.

Open Access Statement: This is an Open Access article distributed in accordance with the Creative Commons Attribution-NonCommercial-NoDerivs 4.0 International License (CC BY-NC-ND 4.0), which permits the noncommercial replication and distribution of the article with the strict proviso that no changes or edits are made and the original work is properly cited (including links to both the formal publication through the relevant DOI and the license). See: https://creativecommons.org/licenses/by-nc-nd/4.0/.

\section{References}

1. Noone AM HN, Krapcho M, Miller D, Brest A, Yu M, Ruhl J, Tatalovich Z, Mariotto A, Lewis DR, Chen HS, Feuer EJ, Cronin KA (eds). SEER Cancer Statistics
Review, 1975-2015 Bethesda, MD: National Cancer Institute; 2018 [updated 2017 Nov; cited 20201 Jun]. Available online: https://seer.cancer.gov/archive/ csr/1975_2015.

2. Walter FM, Rubin G, Bankhead C, et al. Symptoms and other factors associated with time to diagnosis and stage of lung cancer: a prospective cohort study. Br J Cancer 2015;112 Suppl 1:S6-13.

3. Ost D, Fein AM, Feinsilver SH. Clinical practice. The solitary pulmonary nodule. $\mathrm{N}$ Engl J Med 2003;348:2535-42.

4. Vachani A, Tanner NT, Aggarwal J, et al. Factors that influence physician decision making for indeterminate pulmonary nodules. Ann Am Thorac Soc 2014;11:1586-91.

5. Massion PP, Walker RC. Indeterminate pulmonary nodules: risk for having or for developing lung cancer? Cancer Prev Res (Phila) 2014;7:1173-8.

6. National Lung Screening Trial Research Team, Aberle DR, Adams AM, et al. Reduced lung-cancer mortality with low-dose computed tomographic screening. N Engl J Med 2011;365:395-409.

7. de Koning HJ, van der Aalst CM, de Jong PA, et al. Reduced Lung-Cancer Mortality with Volume CT Screening in a Randomized Trial. N Engl J Med 2020;382:503-13.

8. Leleu O, Basille D, Auquier M, et al. Lung Cancer Screening by Low-Dose CT Scan: Baseline Results of a French Prospective Study. Clin Lung Cancer 2020;21:145-52.

9. McWilliams A, Tammemagi MC, Mayo JR, et al. Probability of cancer in pulmonary nodules detected on first screening CT. N Engl J Med 2013;369:910-9.

10. Tammemagi MC, Schmidt H, Martel S, et al. Participant selection for lung cancer screening by risk modelling (the Pan-Canadian Early Detection of Lung Cancer [PanCan] study): a single-arm, prospective study. Lancet Oncol 2017;18:1523-31.

11. Gohagan J, Marcus P, Fagerstrom R, et al. Baseline findings of a randomized feasibility trial of lung cancer screening with spiral CT scan vs chest radiograph: the Lung Screening Study of the National Cancer Institute. Chest 2004;126:114-21.

12. Becker N, Motsch E, Gross ML, et al. Randomized Study on Early Detection of Lung Cancer with MSCT in Germany: Results of the First 3 Years of Follow-up After Randomization. J Thorac Oncol 2015;10:890-6.

13. Gould MK, Tang T, Liu IL, et al. Recent Trends in the Identification of Incidental Pulmonary Nodules. Am J 
Respir Crit Care Med 2015;192:1208-14.

14. Veeramachaneni NK, Crabtree TD, Kreisel D, et al. A thoracic surgery clinic dedicated to indeterminate pulmonary nodules: too many scans and too little pathology? J Thorac Cardiovasc Surg 2009;137:30-5.

15. Mortani Barbosa EJ, Jr. Lung cancer screening overdiagnosis: reports of overdiagnosis in screening for lung cancer are grossly exaggerated. Acad Radiol 2015;22:976-82.

16. Bach PB, Mirkin JN, Oliver TK, et al. Benefits and harms of CT screening for lung cancer: a systematic review. JAMA 2012;307:2418-29.

17. Mazzone PJ, Silvestri GA, Patel S, et al. Screening for Lung Cancer: CHEST Guideline and Expert Panel Report. Chest 2018;153:954-85.

18. Wahidi MM, Govert JA, Goudar RK, et al. Evidence for the treatment of patients with pulmonary nodules: when is it lung cancer?: ACCP evidence-based clinical practice guidelines (2nd edition). Chest 2007;132:94S-107S.

19. Garcia-Velloso MJ, Bastarrika G, de-Torres JP, et al. Assessment of indeterminate pulmonary nodules detected in lung cancer screening: Diagnostic accuracy of FDG PET/CT. Lung Cancer 2016;97:81-6.

20. Ru Zhao Y, Xie X, de Koning HJ, et al. NELSON lung cancer screening study. Cancer Imaging 2011;11 Spec No A:S79-84.

21. Horeweg N, van Rosmalen J, Heuvelmans MA, et al. Lung cancer probability in patients with CT-detected pulmonary nodules: a prespecified analysis of data from the NELSON trial of low-dose CT screening. Lancet Oncol 2014;15:1332-41.

22. Larici AR, Farchione A, Franchi P, et al. Lung nodules: size still matters. Eur Respir Rev 2017;26:170025.

23. National Lung Screening Trial Research Team, Church TR, Black WC, et al. Results of initial low-dose computed tomographic screening for lung cancer. N Engl J Med 2013;368:1980-91.

24. Sánchez M, Benegas M, Vollmer I. Management of incidental lung nodules $<8 \mathrm{~mm}$ in diameter. J Thorac Dis 2018;10:S2611-27.

25. Lee KH, Goo JM, Park SJ, et al. Correlation between the size of the solid component on thin-section CT and the invasive component on pathology in small lung adenocarcinomas manifesting as ground-glass nodules. J Thorac Oncol 2014;9:74-82.

26. O JH, Yoo Ie R, Kim SH, et al. Clinical significance of small pulmonary nodules with little or no $18 \mathrm{~F}-\mathrm{FDG}$ uptake on PET/CT images of patients with nonthoracic malignancies. J Nucl Med 2007;48:15-21.

27. Wang X, Leader JK, Wang R, et al. Vasculature surrounding a nodule: A novel lung cancer biomarker. Lung Cancer 2017;114:38-43.

28. Chen XB, Yan RY, Zhao K, et al. Nomogram For The Prediction Of Malignancy In Small (8-20 mm) Indeterminate Solid Solitary Pulmonary Nodules In Chinese Populations. Cancer Manag Res 2019;11:9439-48.

29. Borghesi A, Michelini S, Nocivelli G, et al. Solid Indeterminate Pulmonary Nodules Less Than or Equal to $250 \mathrm{~mm}$ (3): Application of the Updated Fleischner Society Guidelines in Clinical Practice. Radiol Res Pract 2019;2019:7218258.

30. Choi Y, Gil BM, Chung MH, et al. Comparing attenuations of malignant and benign solitary pulmonary nodule using semi-automated region of interest selection on contrast-enhanced CT. J Thorac Dis 2019;11:2392-401.

31. Li M, Narayan V, Gill RR, et al. Computer-Aided Diagnosis of Ground-Glass Opacity Nodules Using OpenSource Software for Quantifying Tumor Heterogeneity. AJR Am J Roentgenol 2017;209:1216-27.

32. Callister ME, Baldwin DR, Akram AR, et al. British Thoracic Society guidelines for the investigation and management of pulmonary nodules. Thorax 2015;70 Suppl 2:ii1-ii54.

33. Borghesi A, Michelini S, Scrimieri A, et al. Solid Indeterminate Pulmonary Nodules of Less Than 300 mm3: Application of Different Volume Doubling Time Cut-offs in Clinical Practice. Diagnostics (Basel) 2019;9:62.

34. Digumarthy SR, Padole AM, Rastogi S, et al. Predicting malignant potential of subsolid nodules: can radiomics preempt longitudinal follow up CT? Cancer Imaging 2019;19:36.

35. Walker R, Deppen S, Smith G, et al. 68Ga-DOTATATE PET/CT imaging of indeterminate pulmonary nodules and lung cancer. PLoS One 2017;12:e0171301.

36. Perandini S, Soardi GA, Motton M, et al. Solid pulmonary nodule risk assessment and decision analysis: comparison of four prediction models in 285 cases. Eur Radiol 2016;26:3071-6.

37. Swensen SJ, Silverstein MD, Ilstrup DM, et al. The probability of malignancy in solitary pulmonary nodules. Application to small radiologically indeterminate nodules. Arch Intern Med 1997;157:849-55.

38. Mery CM, Pappas AN, Bueno R, et al. Relationship between a history of antecedent cancer and the probability of malignancy for a solitary pulmonary nodule. Chest 
2004;125:2175-81.

39. Surgery AAfT. Lung Cancer Risk Assessment Tool [cited 20201 Jun]. Available online: https://www.aats.org/ aatsimis/AATSWeb/Association/About/Resources/Lung_ Cancer_Risk_Assessment_Tool.

40. Gould MK, Fletcher J, Iannettoni MD, et al. Evaluation of patients with pulmonary nodules: when is it lung cancer?: ACCP evidence-based clinical practice guidelines (2nd edition). Chest 2007;132:108S-30S.

41. Maiga AW, Deppen SA, Massion PP, et al. Communication About the Probability of Cancer in Indeterminate Pulmonary Nodules. JAMA Surg 2018;153:353-7.

42. Massion PP, Healey GF, Peek LJ, et al. Autoantibody Signature Enhances the Positive Predictive Power of Computed Tomography and Nodule-Based Risk Models for Detection of Lung Cancer. J Thorac Oncol 2017;12:578-84.

43. Mitchell PS, Parkin RK, Kroh EM, et al. Circulating microRNAs as stable blood-based markers for cancer detection. Proc Natl Acad Sci U S A 2008;105:10513-8.

44. Lin $Y$, Leng Q, Jiang Z, et al. A classifier integrating plasma biomarkers and radiological characteristics for distinguishing malignant from benign pulmonary nodules. Int J Cancer 2017;141:1240-8.

45. Xing L, Su J, Guarnera MA, et al. Sputum microRNA biomarkers for identifying lung cancer in indeterminate solitary pulmonary nodules. Clin Cancer Res 2015;21:484-9.

46. Ma J, Lin Y, Zhan M, et al. Differential miRNA expressions in peripheral blood mononuclear cells for diagnosis of lung cancer. Lab Invest 2015;95:1197-206.

47. Ma J, Guarnera MA, Zhou W, et al. A Prediction Model Based on Biomarkers and Clinical Characteristics for Detection of Lung Cancer in Pulmonary Nodules. Transl Oncol 2017;10:40-5.

48. Seder CW, Kubasiak JC, Pithadia R, et al. Angiogenesis Biomarkers May Be Useful in the Management of Patients With Indeterminate Pulmonary Nodules. Ann Thorac Surg 2015;100:429-36.

49. Li XJ, Hayward C, Fong PY, et al. A blood-based proteomic classifier for the molecular characterization of pulmonary nodules. Sci Transl Med 2013;5:207ra142.

50. Vachani A, Hammoud Z, Springmeyer S, et al. Clinical Utility of a Plasma Protein Classifier for Indeterminate Lung Nodules. Lung 2015;193:1023-7.

51. Lastwika KJ, Kargl J, Zhang Y, et al. Tumor-derived Autoantibodies Identify Malignant Pulmonary Nodules. Am J Respir Crit Care Med 2019;199:1257-66.
52. Wan JCM, Massie C, Garcia-Corbacho J, et al. Liquid biopsies come of age: towards implementation of circulating tumour DNA. Nat Rev Cancer 2017;17:223-38.

53. Tailor TD, Rao X, Campa MJ, et al. Whole Exome Sequencing of Cell-Free DNA for Early Lung Cancer: A Pilot Study to Differentiate Benign From Malignant CTDetected Pulmonary Lesions. Front Oncol 2019;9:317.

54. Chen C, Huang X, Yin W, et al. Ultrasensitive DNA hypermethylation detection using plasma for early detection of NSCLC: a study in Chinese patients with very small nodules. Clin Epigenetics 2020;12:39.

55. Abbosh C, Birkbak NJ, Wilson GA, et al. Phylogenetic ctDNA analysis depicts early-stage lung cancer evolution. Nature 2017;545:446-51.

56. Kim JI, Park CM, Kim H, et al. Non-specific benign pathological results on transthoracic core-needle biopsy: how to differentiate false-negatives? Eur Radiol 2017;27:3888-95.

57. Witt BL, Cohen MB, Chadwick BE, et al. Cytomorphology is often insufficient to categorize nonsmall-cell lung carcinoma on FNA specimens. Diagn Cytopathol 2016;44:73-9.

58. Munir S, Koppikar S, Hopman WM, et al. Diagnostic Yield for Cancer and Diagnostic Accuracy of Computed Tomography-guided Core Needle Biopsy of Subsolid Pulmonary Lesions. J Thorac Imaging 2017;32:50-6.

59. Lee KH, Lim KY, Suh YJ, et al. Nondiagnostic Percutaneous Transthoracic Needle Biopsy of Lung Lesions: A Multicenter Study of Malignancy Risk. Radiology 2019;290:814-23.

60. Layfield LJ, Dodd L, Witt B. Malignancy risk for the categories: Non-diagnostic, benign, atypical, suspicious, and malignant used in the categorization of endobronchial ultrasound guided-fine needle aspirates of pulmonary nodules. Diagn Cytopathol 2015;43:892-6.

61. Ali MS, Sethi J, Taneja A, et al. Computed Tomography Bronchus Sign and the Diagnostic Yield of Guided Bronchoscopy for Peripheral Pulmonary Lesions. A Systematic Review and Meta-Analysis. Ann Am Thorac Soc 2018;15:978-87.

62. Ng YL, Patsios D, Roberts H, et al. CT-guided percutaneous fine-needle aspiration biopsy of pulmonary nodules measuring $10 \mathrm{~mm}$ or less. Clin Radiol 2008;63:272-7.

63. Wallace MJ, Krishnamurthy S, Broemeling LD, et al. CT-guided percutaneous fine-needle aspiration biopsy of small $(<$ or $=1-\mathrm{cm})$ pulmonary lesions. Radiology 2002;225:823-8. 
64. Hwang HS, Chung MJ, Lee JW, et al. C-arm conebeam CT-guided percutaneous transthoracic lung biopsy: usefulness in evaluation of small pulmonary nodules. AJR Am J Roentgenol 2010;195:W400-7.

65. Choo JY, Park CM, Lee NK, et al. Percutaneous transthoracic needle biopsy of small $(</=1 \mathrm{~cm})$ lung nodules under $\mathrm{C}$-arm cone-beam CT virtual navigation guidance. Eur Radiol 2013;23:712-9.

66. Carillo GA, Vazquez JE, Villar AF. Prevalence of benign pulmonary lesions excised for suspicion of malignancy: could it reflect a quality management index of indeterminate lung lesions? Korean J Thorac Cardiovasc Surg 2014;47:458-64.

67. Howington JA. The role of VATS for staging and diagnosis in patients with non-small cell lung cancer. Semin Thorac Cardiovasc Surg 2007;19:212-6.

68. Ichinose J, Mun M, Matsuura Y, et al. Efficiency of thoracoscopic palpation in localizing small pulmonary nodules. Surg Today 2019;49:921-6.

69. Ko KH, Huang TW, Lee SC, et al. A simple and efficient method to perform preoperative pulmonary nodule localization: CT-guided patent blue dye injection. Clin Imaging 2019;58:74-9.

70. Manca G, Davini F, Tardelli E, et al. Clinical Impact of Radioguided Localization in the Treatment of Solitary Pulmonary Nodule: A 20-Year Retrospective Analysis. Clin Nucl Med 2018;43:317-22.

71. Gill RR, Zheng Y, Barlow JS, et al. Image-guided video assisted thoracoscopic surgery (iVATS) - phase I-II clinical trial. J Surg Oncol 2015;112:18-25.

doi: $10.21037 /$ ccts-20-113

Cite this article as: Wadowski B, Bueno R. Narrative review: indeterminate pulmonary nodules. Curr Chall Thorac Surg $2022 ; 4: 39$.
72. Gill RR, Barlow J, Jaklitsch MT, et al. Image-guided videoassisted thoracoscopic resection (iVATS): Translation to clinical practice-real-world experience. J Surg Oncol 2020;121:1225-32.

73. Yu PSY, Man Chu C, Lau RWH, et al. Video-assisted thoracic surgery for tiny pulmonary nodules with realtime image guidance in the hybrid theatre: the initial experience. J Thorac Dis 2018;10:2933-9.

74. Isaka T, Takahashi K, Maehara T, et al. Intraoperative core needle biopsy under complete video-assisted thoracic surgery for indeterminate tumor of lung. Surg Endosc 2015;29:3579-87.

75. Kennedy GT, Okusanya OT, Keating JJ, et al. The Optical Biopsy: A Novel Technique for Rapid Intraoperative Diagnosis of Primary Pulmonary Adenocarcinomas. Ann Surg 2015;262:602-9.

76. Murphy SJ, Harris FR, Kosari F, et al. Using Genomics to Differentiate Multiple Primaries From Metastatic Lung Cancer. J Thorac Oncol 2019;14:1567-82.

77. Gordon GJ, Deters LA, Nitz MD, et al. Differential diagnosis of solitary lung nodules with gene expression ratios. J Thorac Cardiovasc Surg 2006;132:621-7.

78. De Rienzo A, Yeap BY, Cibas ES, et al. Gene expression ratio test distinguishes normal lung from lung tumors in solid tissue and FNA biopsies. J Mol Diagn 2014;16:267-72.

79. Christensen BC, Marsit CJ, Houseman EA, et al. Differentiation of lung adenocarcinoma, pleural mesothelioma, and nonmalignant pulmonary tissues using DNA methylation profiles. Cancer Res 2009;69:6315-21. 